\title{
Host strain specific sex pheromone variation in Spodoptera
} frugiperda Astrid T Groot*1, Melanie Marr ${ }^{1}$, Gerhard Schöfl1', Sybille Lorenz², Ales Svatos ${ }^{2}$ and David G Heckel ${ }^{1}$

\author{
Address: ${ }^{1}$ Max Planck Institute for Chemical Ecology, Dept. Entomology, Hans-Knöll Strasse 8, 07745 Jena, Germany and ${ }^{2}$ Max Planck Institute \\ for Chemical Ecology, Research group Mass spectrometry, Hans-Knöll Strasse 8, 07745 Jena, Germany \\ Email: Astrid T Groot* - agroot@ice.mpg.de; Melanie Marr - mmarr@ice.mpg.de; Gerhard Schöfl - gschoefl@ice.mpg.de; \\ Sybille Lorenz - lorenz@ice.mpg.de; Ales Svatos - svatos@ice.mpg.de; David G Heckel - heckel@ice.mpg.de \\ * Corresponding author
}

Published: 25 December 2008

Frontiers in Zoology 2008, 5:20 doi:10.1186/1742-9994-5-20
Received: 17 September 2008

Accepted: 25 December 2008

This article is available from: http://www.frontiersinzoology.com/content/5///20

(C) 2008 Groot et al; licensee BioMed Central Ltd.

This is an Open Access article distributed under the terms of the Creative Commons Attribution License (http://creativecommons.org/licenses/by/2.0), which permits unrestricted use, distribution, and reproduction in any medium, provided the original work is properly cited.

\begin{abstract}
Background: The fall armyworm Spodoptera frugiperda (Lepidoptera; Noctuidae) consists of two distinct strains with different host plant preferences for corn and rice. To assess whether pheromonal-mediated behavioral isolation accompanies the habitat isolation on different host plants, we compared the sex pheromone composition among females of the two strains. Pheromone glands were extracted with or without injection of pheromone biosynthesis activating neuropeptide (PBAN). To assess the mode of inheritance of this variation, we also analyzed the pheromone composition of $F_{1}$ hybrid females.
\end{abstract}

Results: Relative to intra-strain variation, the pheromone composition of the two strains differed significantly. Corn strain females contained significantly more of the second most abundant pheromone compound ZII-I6:Ac (m), and significantly less of most other compounds, than rice strain females. When females were injected with PBAN before their glands were extracted, the differences between the strains were less pronounced but still statistically significant. The pheromone composition of hybrid females showed a maternal inheritance of the major component Z9-I 4:Ac (M) as well as of ZII-16:Ac (m). Most other compounds showed an inheritance indicating genetic dominance of the corn strain. The within-strain phenotypic correlations among the various components were consistent with their hypothesized biosynthetic pathway, and between-strain differences in the correlation structure suggested candidate genes that may explain the pheromone differences between the two strains. These include $\Delta 9$ - and $\Delta \mathrm{II}$ desaturases, and possibly also a $\Delta 7$-desaturase, although the latter has not been identified in insects so far.

Conclusion: The two host strains of $S$. frugiperda produce systematically differing female sex pheromone blends. Previously-documented geographic variation in the sexual communication of this species did not take strain identity into account, and thus may be partly explained by different strain occurrence in different regions. The finding of pheromone differences reinforces the possibility of incipient reproductive isolation among these strains, previously shown to differ in the timing of nocturnal mating activity and host plant use. Finding the genetic basis of the pheromone differences, as well as these other biological traits, will help to elucidate the role of premating isolation in the continuing differentiation of these two strains that may eventually lead to speciation. 


\section{Background}

In night-flying moths, highly specific, long distance, pheromonal communication ensures that males and females can find each other and mate. Females produce a speciesspecific sex pheromone in a specialized gland at the tip of their abdomen, to which males of the same species are attracted [1,2]. Moth pheromones usually consist of a blend of two or more components of even-numbered $\mathrm{C}_{10}-\mathrm{C}_{18}$ straight-chain, unsaturated derivatives of fatty acids, with the carbonyl carbon modified to form an oxygen-containing functional group (alcohol, aldehyde, or acetate ester) $[3,4]$. The species-specificity of each blend is determined by the particular combination of the components, as well as their relative ratios (e.g., [5-7]). Although there are thousands of moth species with unique pheromone blends (e.g., [8]), the evolutionary processes that resulted in this diversity of sexual communication signals are still poorly understood (e.g., [9-11]). To gain insight into the evolution of premating isolation between species it is essential to quantify the level and possible causes of variation in the premating signals within species on which selection may operate.

The fall armyworm (FAW, Spodoptera frugiperda J. E. Smith) offers an ideal opportunity for investigating intraspecific variation in sex pheromone communication, because two sympatrically occurring strains have already been recognized $[12,13]$. One feeds predominantly on corn (the corn strain, $\mathrm{C}$ ) and the other on rice and various pasture grasses (the rice strain, $\mathrm{R}$ ). These strains can be identified by several molecular markers, e.g. allele frequency differences at three allozyme markers [12,14], several strain specific DNA sequence variants in the mitochondrial COI gene [15,16] and ND1 gene [17], AFLP markers $[18,19]$, and the FR repetitive nuclear DNA sequence extensively present in R and mostly absent from $\mathrm{C}[14,20]$. Co-occurrence of typically strain-specific markers in the same individuals has provided evidence of some naturally-occurring hybridization in the field [18].

Given that natural hybridization can occur, factors that might limit it and thus maintain the genetic integrity of two different strains are of interest. The two strains differ in the timing of their mating activity; female calling (emission of pheromone) and mating occurs early in the night for $\mathrm{C}$ and during the last half of the night for $\mathrm{R}[21,22]$. When using live females as lures in pheromone traps and typing a subset of males caught in these traps, significantly more $\mathrm{C}$ males were caught in traps with $\mathrm{C}$ females, and $\mathrm{R}$ females attracted more $\mathrm{R}$ males [21]. Under laboratory conditions, we have found that both strains mate assortatively to some degree as well (G. Schöfl, A. Dill, A.T. Groot, unpubl. res.), which would inhibit hybridization and enhance divergence between the strains.

The female sex pheromone composition and male attraction in the field have been studied in several regions within the North and South American range of $S$. frugiperda (see Table 1). The female pheromone glands were found to contain Z9-14:Ac as the major compound (to which we will refer as $M$ ), comprising up to $83 \%$, as well as the second most abundant compound Z11-16:Ac (m), and a number of compounds present in low amounts, such as Z9-12:Ac and Z7-12:Ac (see Table 1) [23-25]. In addition, Brazilian $S$. frugiperda females produce E7-12:Ac [25], a compound not found in other populations so far.

Table I: Means and Coefficients of variation (CV) of each compound when 7 compounds and when 4 compounds of the sex pheromone gland of Spodoptera frugiperda are considered.

\begin{tabular}{|c|c|c|c|c|c|c|c|c|c|c|c|c|}
\hline \multirow[b]{2}{*}{7 Compounds } & \multicolumn{2}{|c|}{$\begin{array}{c}\text { Corn }-\mathrm{Sc}^{1} \\
(\mathrm{n}=17)\end{array}$} & \multicolumn{2}{|c|}{$\begin{array}{l}\text { Rice }-S c^{\prime} \\
(n=22)\end{array}$} & \multicolumn{2}{|c|}{$\begin{array}{c}\text { Corn }{ }^{2} \\
(n=76)\end{array}$} & \multicolumn{2}{|c|}{$\begin{array}{l}C \times R^{2} \\
(n=75\end{array}$} & \multicolumn{2}{|c|}{$\begin{array}{l}R \times C^{2} \\
(n=74)\end{array}$} & \multicolumn{2}{|c|}{$\begin{array}{c}\text { Rice }^{2} \\
(n=59)\end{array}$} \\
\hline & Mean & $C V$ & Mean & $\mathrm{CV}$ & Mean & $\mathrm{CV}$ & Mean & $\mathrm{CV}$ & Mean & $C V$ & Mean & $\mathrm{CV}$ \\
\hline ZII-I6:Ac (m) & 12.4 & 50 & 7.3 & 50 & 12.5 & 33 & 13.9 & 33 & 10.0 & 24 & 9.3 & 33 \\
\hline Z9-I4:Ac (M) & 81.8 & 7 & 82.4 & 8 & 83.5 & 5.5 & 82.3 & 6 & 86.1 & 5 & 84.0 & 5 \\
\hline I4:Ac (a) & 1.1 & 65 & 1.1 & 59 & 1.0 & 117 & 0.9 & 52 & 0.9 & 113 & 1.5 & 62 \\
\hline ZII-I4:Ac (b) & I.I & 35 & 1.6 & 55 & 1.0 & 69 & 1.0 & 40 & 1.0 & 61 & 1.5 & 41 \\
\hline I2:Ac (c) & I.I & 42 & 2.0 & 44 & 0.4 & 53 & 0.5 & 51 & 0.7 & 180 & 1.0 & 81 \\
\hline Z9-12:Ac (d) & 0.8 & 52 & 2.1 & 55 & 0.7 & 89 & 0.7 & 77 & 0.6 & 70 & 1.5 & 86 \\
\hline Z7-I2:Ac (e) & 1.8 & 48 & 3.6 & 47 & 0.9 & 45 & 0.8 & 54 & 0.7 & 37 & I.I & 51 \\
\hline \multicolumn{13}{|l|}{4 Compounds } \\
\hline ZII-I6:Ac (m) & 12.8 & 50 & 7.7 & 51 & 12.8 & 33 & 14.3 & 33 & 10.3 & 25 & 9.7 & 33 \\
\hline Z9-I4:Ac (M) & 84.6 & 7 & 86.3 & 6 & 85.6 & 5 & 84.2 & 5 & 88.3 & 3 & 87.6 & 4 \\
\hline Z9-I2:Ac (d) & 0.8 & 53 & 2.3 & 58 & 0.7 & 97 & 0.7 & 78 & 0.6 & 77 & 1.6 & 89 \\
\hline Z7-I2:Ac (e) & 1.8 & 49 & 3.8 & 48 & 1.0 & 45 & 0.8 & 54 & 0.8 & 39 & 1.1 & 53 \\
\hline
\end{tabular}

IGlands extracted in the scotophase; ${ }^{2}$ Glands extracted from PBAN-injected females 
Not all compounds that are found in the sex pheromone glands of females are "pheromone components" which are by definition important in the attraction of conspecific males (e.g. [6]; some may be unavoidable by-products of the pheromone biosynthetic pathways [4]. The components that have been found to be attractive for $S$. frugiperda males include the major component Z9-14:Ac (M), as well as one of the components present in only low amounts, Z7-12:Ac (to which we refer as e). This is the crucial secondary pheromone component of $S$. frugiperda, because blends without it are not attractive for males of this species [23-29]. The addition of other components has given different results in different regions, which suggests that the sexual communication of this species varies geographically [25], similar to what has been found in several other noctuid moth species (e.g., [30-32]. Specifically, when Z11-16:Ac (m) was added to the blend, significantly more males were attracted in Mexico and Costa Rica [26,27], but not in Florida [23]. In Pennsylvania, adding Z11-16:Ac (m) as well as Z9-12:Ac (d) to the twocomponent blend attracted twice as many males as the two-component blend [29]. By contrast, in Brazil the addition of Z11-16:Ac to the two-component blend did not increase attraction of $S$. frugiperda males, but the addition of E7-12:Ac, that was found uniquely in glands of Brazilian females, did [25].

Surprisingly, none of the above-described studies mention whether corn or rice strain females were analyzed or whether corn or rice strain males were attracted to the different blends. This is despite the documentation of both strains in North America $[12,13,15,21]$ as well as in Brazil [33]. Because of the lack of distinction between the two strains in previous pheromone studies, the variation found in the different regions could be at least partly due to sampling of two different strains in the different areas.

Another possible source of variation found in the pheromone composition is the time of day at which glands are extracted. Female moths usually produce pheromone de novo every night [34], and in many species the timing of pheromone synthesis and release is controlled by Pheromone Biosynthesis Activating Neuropeptide (PBAN) [35]. Extraction of glands after dark (i.e. in the scotophase) yields those compounds that have accumulated there in response to natural PBAN produced in the suboesophagal ganglion and released from the corpus cardiacum into the hemolymph [36,37]. Experimental injection of commercially available PBAN induces pheromone production within 2-3 hours, independent of the time of day or the physiological state (mating status and age) of the female [35,38-41]. This thus can exclude the time of day as a possible source of variation, which is important in the two strains of $S$. frugiperda, because they differ in their time of mating activity and thus likely also the time of pheromone production at night. Previously, we found that injecting females with PBAN can be a simple and convenient method of determining a female's native pheromone phenotype [42]. However, PBAN can act at different stages in the pheromone biosynthetic pathway in different species, reviewed by $[4,41]$. Therefore, it is important to assess the effect of PBAN injections on the pheromone composition in the two strains.

The aim of our study was to determine whether the pheromone composition in the glands of corn strain females differs from that of rice strain females. We analyzed the pheromone composition of $F_{1}$ hybrid females as well, to assess the mode of inheritance of pheromone variation. We found signficant differences in pheromone composition between corn and rice strain females. Although PBAN reduced the variation in both strains, the pheromone composition remained significantly different. The relative amounts of the two most abundant components, Z914:Ac $(M)$ and Z11-16:Ac (m), were maternally inherited, while three minor compounds were inherited in a manner indicating genetic dominance of alleles from the corn strain. Linking these differences to the phenotypic correlations between the pheromone compounds, as well as to a hypothetical scheme of the biosynthetic pathway of the pheromone of this species, suggests that differential activity of a $\Delta 11-, \Delta 9-$, and/or possibly also a $\Delta 7$-desaturase may underly the pheromone variation between the two strains.

\section{Results}

\section{Between-strain pheromone variation}

Comparing the gland content between corn and rice strain females that had been extracted under natural conditions in the scotophase, we found a significant overall difference in the pheromone composition as determined by the relative amounts of most of the minor compounds (Fig. 1; see Table 1 for Coefficients of Variation). Corn strain females contained significantly more Z11-16:Ac (m) and significantly less 12:Ac (c), Z9-12:Ac (d), and Z7-12:Ac (e) than rice strain females. The relative amount of the major component Z9-14:Ac (M) was the same in the two strains, as well as the relative amount of 14:Ac (a) and Z11-14:Ac (b).

\section{Effect of PBAN on pheromone composition}

In both strains, glands of females injected with PBAN differed significantly in their pheromone composition from glands of females that were extracted in the scotophase (Fig. 2a and 2b). In both strains, significantly more Z1116 :Ac (m) and significantly less of all other minor compounds (a-e) were found in glands of PBAN-injected females. There were no differences between the glands of rice strain females injected with different amounts of PBAN $(1,7.5$ or 20 pmol) (Fig. 2b). Despite the increase 


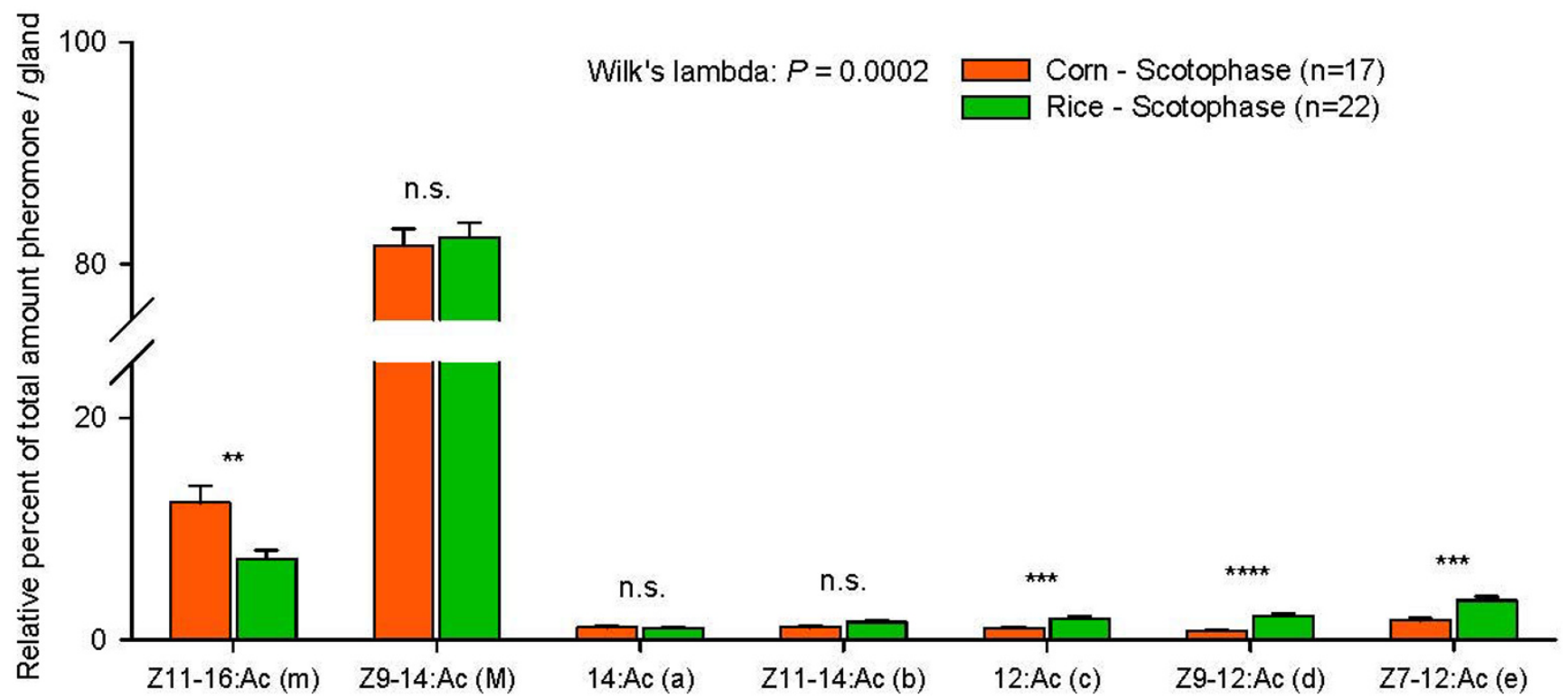

Figure I

Between-strain comparisons of the pheromone composition when glands were extracted from females under natural conditions, i.e. 4-6 $\mathbf{~}$ into scotophase. The total percent of all depicted compounds add to $100 \%$. N.s: not significant, $*$ indicates $P<0.05$, ** indicates $P<0.01$, *** indicates $P<0.001$, **** indicates $P<0.000 \mathrm{I}$.

of Z11-16:Ac (m) and a reduction of most compounds in both strains, the overall pheromone composition still differed significantly between the two strains after injection with 7.5 pmol PBAN (Fig. 2c). Specifically, there was still significantly more Z11-16:Ac (m) and significantly less Z9-12:Ac (d) in corn strain than in rice strain females, although differences in the relative amounts of 12:Ac (c) and Z7-12:Ac (e) were no longer significant.

\section{Mode of inheritance of pheromone variation}

The hybrid CxR females (offspring of $\mathrm{C}$ females and $\mathrm{R}$ males) contained similar amounts of the major component Z9-14:Ac (M) as the corn and the rice strain females, while the reciprocal cross $\mathrm{RxC}$ females contained significantly more of this component than the other three groups (Fig. 3; see Table 1 for Coefficients of Variation). CxR females contained similar relative amounts of Z1116:Ac $(\mathrm{m})$ as corn strain females, while RxC females contained similar relative amounts of Z11-16:Ac (m) as rice strain females. This suggests a maternal inheritance of the relative amount of this component. All other compounds were present in similar amounts in both CxR females and RxC females. For 14:Ac (a), Z11-14:Ac (b), and Z9-12:Ac (d), the relative amounts in the hybrids were similar to corn strain females and significantly lower than rice strain females, suggesting genetic dominance of alleles from the corn strain in the production of these compounds in the hybrids. The remaining two compounds 12:Ac (c) and Z712:Ac (e) showed a different pattern: RxC females con- tained significantly more 12:Ac than corn strain females, and both hybrids contained significantly less Z7-12:AC than the two parental strains.

Not all compounds that we identified from the female glands may be pheromone components functioning to attract males. From the field studies conducted so far, at least the four components Z9-14:Ac (M), Z11-16:Ac (m), Z9-12:Ac (d), and Z7-12:Ac (e) have been shown to affect the attraction of $S$. frugiperda males in one or more populations (see Table 2). The possible attractive role of the other compounds remains to be investigated. When we omitted these other compounds and based our analysis only on the four components that are known to affect the attraction of conspecific males (i.e. the total amount of these four components was set to $100 \%$, after which the relative percentages of each of the four components were recalculated), we also found a significant overall difference in pheromone composition between the corn and the rice strain (see Fig. 4). Specifically, corn strain females contained significantly more Z11-16:Ac (m) and significantly less Z9-12:Ac (d) than rice strain females in all comparisons. The major component Z9-14:Ac (M) was only significantly different between corn and rice strain females when injected with 7.5 pmol PBAN (Fig. 4b). The hybrid females contained similar relative amounts of Z914:Ac (M) and Z11-16:Ac (m) as their mothers, indicating a maternal inheritance of the relative amount these main components. Hybrid females of both types contained sim- 

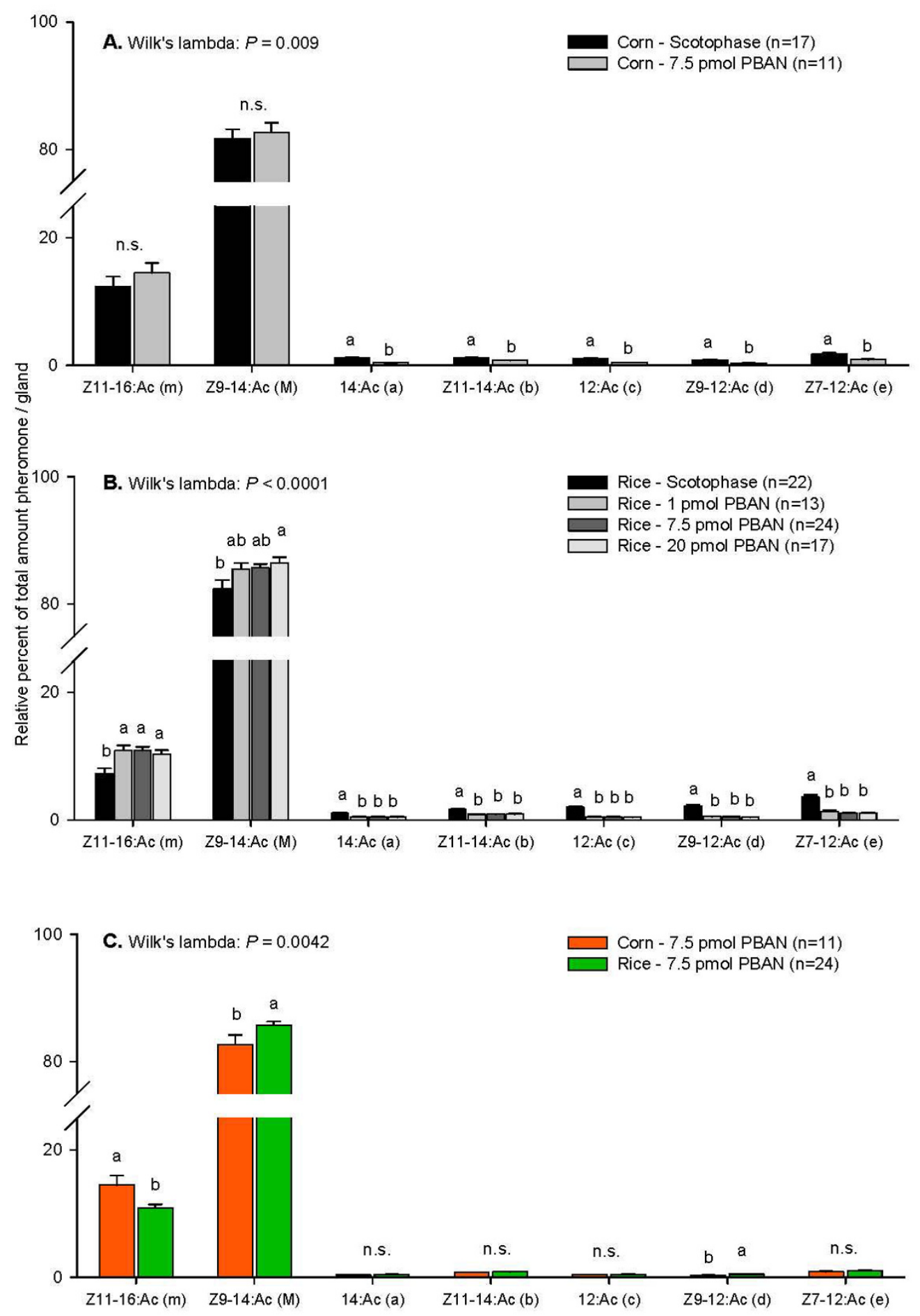

Figure 2

Effect of PBAN on the pheromone composition in the females of the two strains. Glands that were extracted from females in the scotophase were compared to glands that were extracted from females that had been injected with PBAN. A. Glands from corn strain females. B. Glands from rice strain females. C. Comparsion between corn and rice strain glands that were extracted from females that had been injected with 7.5 pmol PBAN. In each graph the total percent of all depicted compounds add to $100 \%$. Different letters above the bars of one component indicate significant differences. N.s: not significant. 


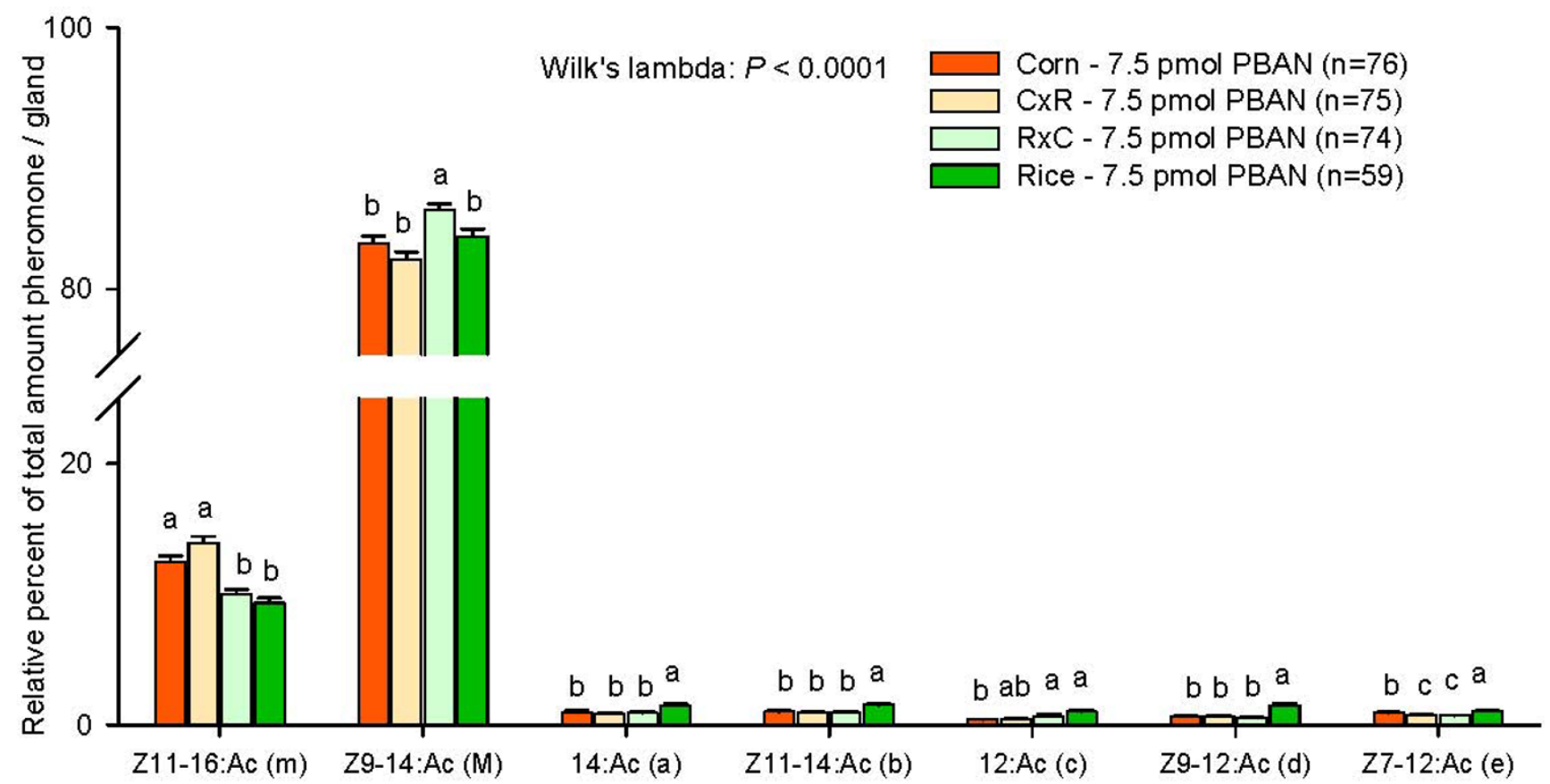

\section{Figure 3}

Mode of inheritance of the pheromone composition. Gland extracts from offspring of corn females mated with rice males $(\mathrm{C} \times \mathrm{R})$ and offspring of rice females mated with corn males $(\mathrm{R} \times \mathrm{C})$ were compared with gland extracts from corn and rice strain females. All females have been injected with 7.5 pmol PBAN before gland extractions. The total percent of all depicted compounds add to $100 \%$. Different letters above the bars of one component indicate significant differences. N.s: not significant.

ilar amounts of Z9-12:Ac (d) as corn strain females, similar to what was found when including all pheromone compounds (Fig. 3). The critical secondary sex pheromone component Z7-12:Ac (e) was significantly higher in scotophase-extracted rice strain females than in corn strain females, but did not differ between PBAN-injected corn and rice strain females. This compound was significantly lower in hybrid females.

\section{Phenotypic correlations}

The previous comparisons have been based on mean proportions of pheromone gland compounds of different groups of females. The proportions within a particular group of females vary slightly from individual to individual around these mean values. The phenotypic correlation matrix describes whether this variation in different components is positively or negatively correlated, or uncorrelated (see Additional files 1, 2, 3). Because the compounds are not produced independently of one another, but are connected due to their biochemical pathways (see Fig. 5), the pattern of correlations may reveal properties of those pathways. A pattern seen within all groups of females tested consisted of strong negative correlations between the major component Z9-14:Ac (M) and both Z11-16:Ac (m) and 14:Ac (a) (orange cells in
Additional files 1, 2, 3). Thus, for all groups of females, when more Z9-14:Ac is produced, less Z11-16:Ac and 14:Ac are produced and vice versa. Also, in all groups of females the minor compounds b-e were mostly positively correlated with each other (green cells in Additional files $1,2,3)$. Another pattern seen within all groups of females, except in corn females whose glands were extracted in the scotophase (Additional file 1a), is the strong positive correlations between 14:Ac (a) and the other minor compounds (b-e), and the strong negative correlations between $M$ and b-e (yellow cells in Additional files 1, 2, 3 ). The most striking difference between PBAN-injected corn females and CxR hybrid females on the one hand, and the other groups of females on the other hand, is the positive correlation between the major component $M$ and the critical secondary sex pheromone component $e$ in the first two groups (blue cell in Additional file $2 a$ and $3 a$ ). In scotophase-extracted corn females and in $\mathrm{RxC}$ females this correlation is absent, while in the other groups of female this correlation is negative.

\section{Discussion}

The relative amounts of the compounds present in the sex pheromone glands of our laboratory-reared corn and rice strain of $S$. frugiperda were significantly different. Rice 
Table 2: Reported sex pheromone components of S. frugiperda

\begin{tabular}{|c|c|c|c|c|c|c|c|c|c|c|c|}
\hline \multicolumn{6}{|c|}{ Female sex pheromone } & \multicolumn{6}{|c|}{ Synthetic lures that attracted most males } \\
\hline & $\mathrm{FI}, \mathrm{USA}^{\mathrm{I}}$ & $\begin{array}{l}\text { French } \\
\text { Guyana }^{2}\end{array}$ & Brazil $^{3}$ & Corn* & Rice* & PA, USA ${ }^{4}$ & $\mathrm{FI}, * *$ USA ${ }^{1,5}$ & Mexico 6 & Costa Rica ${ }^{7}$ & $\begin{array}{l}\text { French } \\
\text { Guyana }^{2}\end{array}$ & Brazil $^{3}$ \\
\hline $\begin{array}{l}Z|I-| 6: A c \\
(m)\end{array}$ & 9 & 16.66 & 12.9 & 12.4 & 7.3 & 17.69 & & 10.3 & & 15.5 & \\
\hline $\begin{array}{l}Z 9-\mid \text { 4:Ac } \\
\text { (M) }\end{array}$ & 69 & 73.75 & 82.8 & 81.7 & 82.4 & 81.61 & 99.42 & 77.8 & 99.4 & 83 & 98 \\
\hline I4:Ac (a) & & 0.53 & & I.I & I.I & & & & & & \\
\hline $\begin{array}{l}Z I I-I 4: A c \\
\text { (b) }\end{array}$ & & 1.2 & 1.5 & 1.1 & 1.6 & & & & & & \\
\hline I2:Ac (c) & & 0.43 & 0.6 & I.I & 1.9 & & & & & & \\
\hline Z9-I2:Ac (d) & 2 & 0.5 & trace & 0.8 & 2.1 & 0.25 & & & & 0.5 & \\
\hline Z7-I 2:Ac (e) & 4 & 1.12 & 0.8 & 1.8 & 3.6 & 0.45 & 0.58 & 11.9 & 0.6 & 1 & I \\
\hline E7-12:Ac & & & 1.2 & & & & & & & & I \\
\hline Z9-|4:AI & 13 & 3.59 & & & & & & & & & \\
\hline ZI0-I4:Ac & & & 0.3 & & & & & & & & \\
\hline $16: A c$ & & 0.21 & & & & & & & & & \\
\hline $\mathrm{ZII-I6:Al}$ & 3 & & & & & & & & & & \\
\hline
\end{tabular}

All numbers in one column add to $100 \%$. Female sex pheromone refers to gland extracts. Tumlinson et al. (1986) also analyzed the volatile blend emitted from the glands and found 2.6\% ZII-16:Ac, 90.1\% Z9-14:Ac, I.2\% ZII-I4:Ac, I.9\% I2:Ac, 2.2\% Z9-12:Ac, 0.21\% 16:Ac and 3.2\% Z7-I2:Ac. Note that the Z9-14:Al which was found in the gland extracts, was not found in the emitted volatiles. Compounds in bold are the compounds that we could identify in the gland extracts studied here; all of these were found in both strains. Letters behind these compounds are given the facilitate the reading. *Means of the relative amounts that we found in glands extracted from females in the scotophase. ** In FL the same 4-component blend as used by Fleischer et al. (2005) was tested and attracted similar amounts of males as the 2-component blend. $[23] ;{ }^{2}[24] ;{ }^{3}[25] ;{ }^{4}[29] ;{ }^{5}[28]$; ${ }^{6}[27] ;{ }^{7}[26]$.

strain females contained a significantly lower relative amount of Z11-16:Ac (m) and a correspondingly higher amount of most other minor compounds than corn strain females. Connecting these differences to the hypothetical biosynthetic pathway of these compounds (Fig. 5), the differences between the strains could be explained by a reduced activity of the $\Delta 11$ desaturase converting 16:CoA to Z11-16:CoA in the rice strain. This would increase the relative amount of 16:CoA available for chain-shortening to $14: \mathrm{CoA}$, the precursor of the minor compounds (c-e) that were found in higher relative amounts in the rice than in the corn strain.
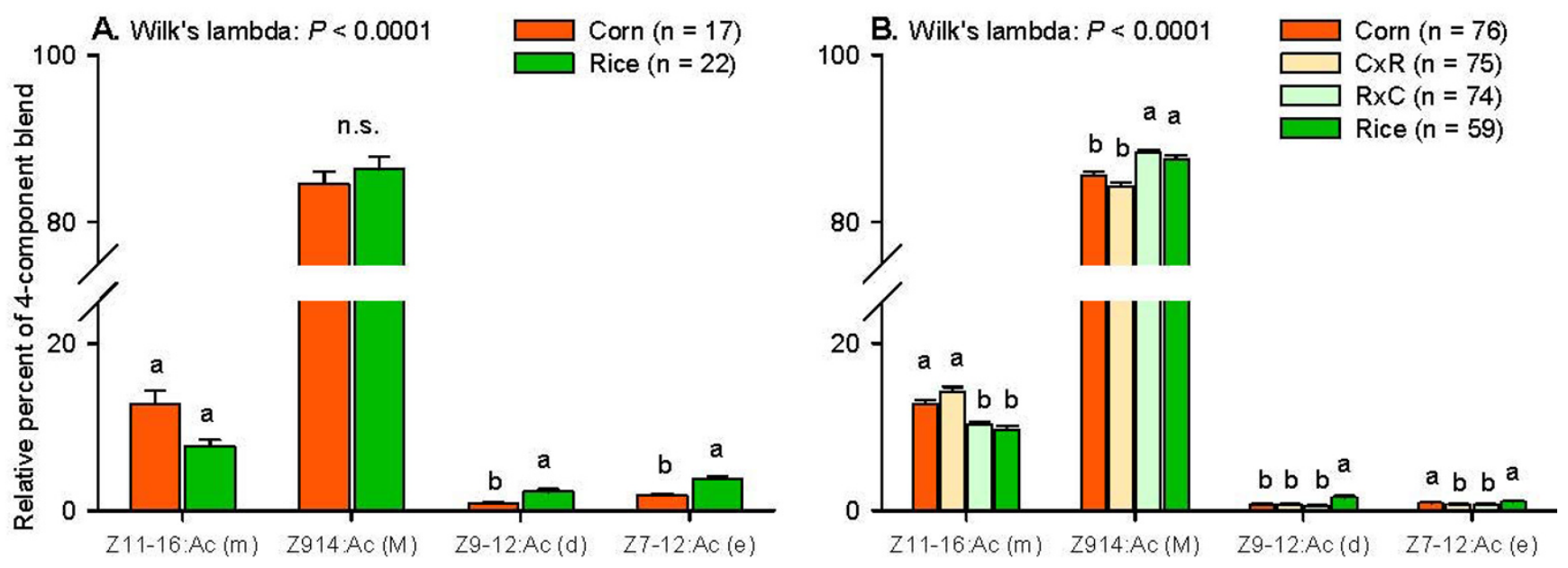

Figure 4

Between-strain comparisons of the pheromone composition between corn and rice strain females, including only the four compounds that have been shown to affect conspecific male attraction. The total percent of the four compounds add to $100 \%$. Different letters above the bars of one component indicate significant differences. N.s: not significant. 


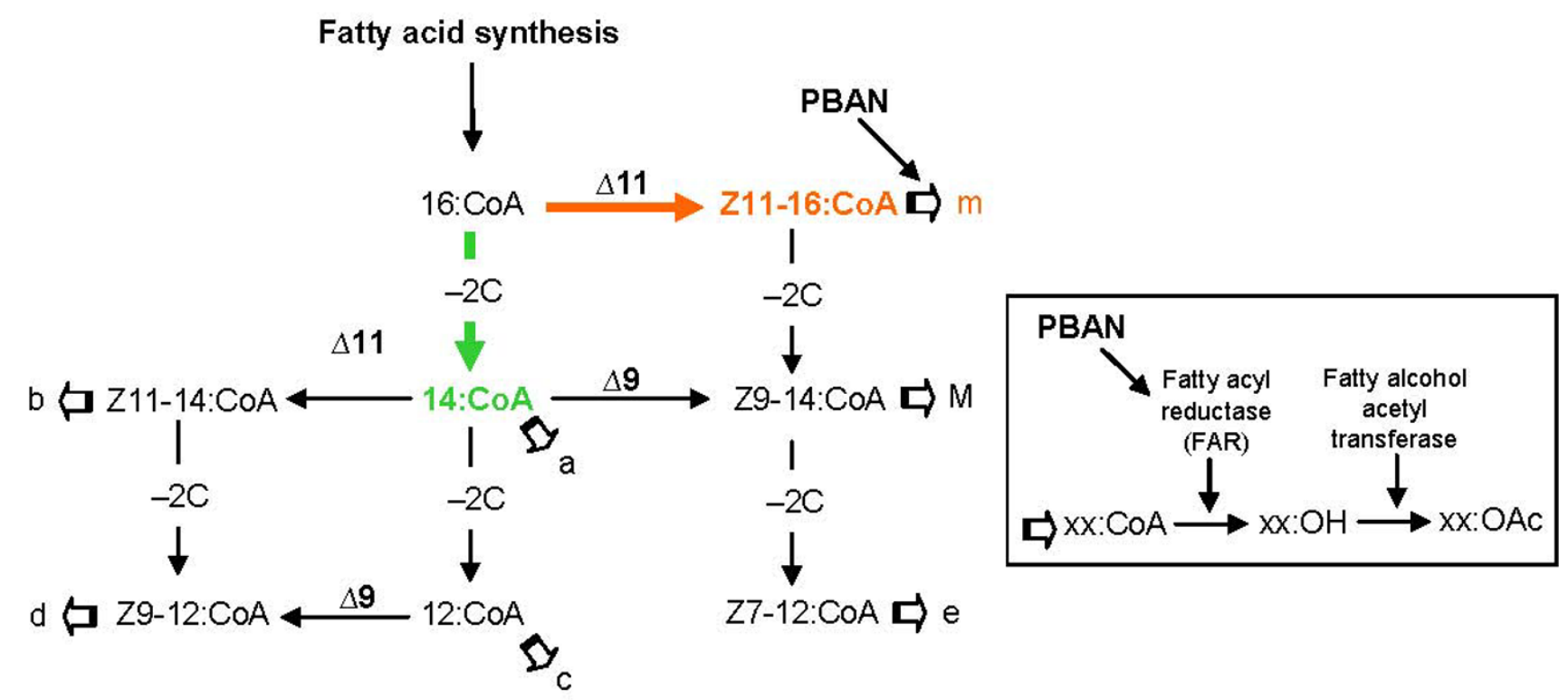

\section{Figure 5}

Proposed pathways of the biosynthesis of the pheromone components in S. frugiperda (based on biosynthetic pathways described for other moth species by Jurenka, 2003). The interplay of desaturation and chain shortening of 16-, and I4-carbon acyl-CoA derivatives produce mono-unsaturated acyl-CoA precursors that are then reduced and acetylated to produce acetate esters. The number that follows $\Delta$ indicates the position of the double bond introduced by the desaturase into the acyl-CoA. $-2 \mathrm{C}$ indicates chain-shortening by two carbons through $\beta$-oxidation. The order of desaturation and chain shortening results in different compounds. The open arrows stand for reduction and acetylation. The letters behind the open arrows stand for the pheromone compounds mentioned in all Tables and Figures.

The biosynthetic scheme can also account for much of the observed correlation structure within groups of females. It is important to note that since $\mathrm{M}$ is the dominant compound, always accounting for more than $80 \%$ of the total, modest variations in its production would have proportionally greater effects on the relative amounts of the minor compounds. Also, an increase in the formation of one product would entail a decrease in the formation of the other. The universal and strong negative correlations between the major component Z9-14:Ac (M) and both Z11-16:Ac (m) and 14:Ac (a) are consistent with the assumption that $\mathrm{Z} 11-16: \mathrm{CoA}$ is the precursor for both $\mathrm{M}$ and $\mathrm{m}$, and that $14: \mathrm{CoA}$ is the precursor of $\mathrm{M}$ and $\mathrm{a}$. Another pattern common to many of the groups is the positive correlation among the four minor compounds b$e$, that all share the same common precursor 14:CoA. If the amount of 14:CoA varies between females within a group, then a larger amount could result in a concomitant increase of all products. The significant positive correlation between the major component Z9-14:Ac (M) and the critical secondary sex pheromone component Z7-12:Ac (e), found only in PBAN-injected corn and CxR females, may be explained by an increased conversion of 16:CoA to Z11-16:CoA coupled to a limited availability of fatty acyl reductase (FAR), so that an excess of Z11-16:CoA is chain-shortened (through $\beta$-oxidation) to Z9-14:CoA and
Z7-12:CoA. Alternatively, PBAN may enhance $\beta$-oxidation in the corn strain.

The finding that injection of PBAN changed the ratio of the pheromone compounds in both the corn and the rice strain suggests that PBAN does not primarily stimulate the synthesis of fatty acid hormone precursors, as in most moth species [43-46], but instead primarily stimulates a step later in the biosynthetic pathway, as in Spodoptera littoralis $[47,48]$. In general, the precursors of moth pheromones are derived from the fatty acid synthesis, which are stearic acid (18:CoA) and palmitic acid (16:CoA). These acids can then be desaturated through $\Delta 11$ - and $\Delta 9$-desaturase, and reduced through $\beta$-oxidation to shorter chain length fatty acids (e.g. 14:CoA, 12:CoA), after which they are reduced and acetylated to form the acetate esters [49]. In $S$. littoralis, PBAN acts on the reduction step of the fatty acids to form the intermediate alcohols $[47,48]$, which is directly followed by the next enzymatic reaction (acetylation) to form the end products [41]. It seems likely that a similar mode of action of PBAN occurs in $S$. frugiperda: after the formation of Z11-16:CoA, PBAN may activate a fatty acyl reductase (FAR), so that $\mathrm{Z} 11-16: \mathrm{OH}$ is formed, which is then converted to Z11-16:Ac (m) (see Fig. 5). Alternatively, PBAN may specifically activate the $\Delta 11$ desaturase that converts the 16 :CoA to Z11-16:CoA. This 
may be a different $\Delta 11$-desaturase than the one converting 14:CoA to Z11-14:CoA, similar to the differential substrate preference found for $\Delta 9$-desaturases [50]. This would explain the significant increase of Z11-16:Ac (m) in glands of PBAN injected females compared to glands of females that were extracted under natural conditions in the scotophase in both strains (see Fig. $2 \mathrm{a}$ and $2 \mathrm{~b}$ ). Despite this effect on the pheromone composition in both strains, decreasing the differences between the two strains, significant differences between the strains were still detectable.

The pheromone composition in hybrid females reveals the mode of inheritance of the pheromone differences between the two strains. The relative amounts of $\mathrm{Z11}$ 16:Ac (m) and probably also 12:Ac (c) in the pheromone glands are maternally inherited. In addition, the major component Z9-14:Ac (M) shows a maternal inheritance as well when only the four components are compared that have been shown to affect the attraction of $S$. frugiperda males (see Fig. 4). The other compounds, 14:Ac (a), Z1114:Ac (b), and Z9-12:Ac (d), are inherited differently, in a corn-dominant way, suggesting that the production of these compounds are affected by a different set of genes. The production of the essential secondary component Z712:Ac (e), without which $S$. frugiperda males are not attracted [23], seems to be suppressed in the hybrid females, as both contained less than either parent. We are currently conducting crosses and backcrosses to identify the genetic basis of all pheromone differences between these two strains.

Candidate genes that may possibly underlie the sex pheromone differences between corn and rice strain females can be found by linking these differences to likely enzymatic differences in the biosynthetic pathway (Fig. 5), as well as by comparing the phenotypic correlations between the pheromone compounds. This is a novel approach that we developed recently [51] to generate a list of candidate genes that may explain pheromone differences between species. The most obvious difference in pheromone composition between the two strains of $S$. frugiperda, i.e. a higher relative amount of Z11-16:Ac (m) in corn than in rice strain females, suggests that a $\Delta 11$-desaturase, converting the 16:CoA to $\mathrm{Z11}-16$ : $\mathrm{CoA}$, is more active in corn than in rice strain females. In addition, the strong positive phenotypic correlation in the rice strain (and $\mathrm{RxC}$ females) between 14:Ac (a) and 12:Ac (c) with Z7-12:Ac (e) suggests a coupling that could be explained by a $\Delta 7$ desaturase. This coupling is absent in corn females, at least when injected with PBAN. Thus, a $\Delta 7$-desaturase, if present, could be restricted to the rice strain. However, so far $\Delta 7$-desaturases have not been identified in insects. An alternative explanation is that $\mathrm{Z7-12:Ac}$ in rice-strain females is produced via a conversion of 14 :CoA by $\Delta 9$ - desaturase to Z9-14:CoA, after which Z9-14:CoA is $\beta$-oxidized to Z7-12:Ac (e). In corn females (and CxR females), the positive correlation beween Z9-14:Ac (M) and Z712:Ac (e), and not between $M$ and 14:Ac, suggests that Z712 :Ac (e) in this strain is produced via $\Delta 11$-desaturation of 16:CoA, which is subsequently $\beta$-oxidized to Z9-14:Ac (M) and Z7-12:Ac (e), as mentioned above. Thus, both $\Delta 11$-desaturase and $\Delta 9$-desaturases, and possibly $\Delta 7$ desaturase, are candidate genes that may be differentially active between the two strains. Desaturases have been identified from pheromone glands of many moth species, e.g., [52]. In the genus Spodoptera, $\Delta 9$ - and $\Delta 11$-desaturases have been characterized in S. exigua, S. littura [52] and $S$. littoralis [53]. These identifications will facilitate the assessment of whether and which of these genes vary between the two strains.

Now that we have found significant differences in the pheromone composition between the two strains, the next steps are to evaluate a) whether different ratios of the pheromone blend are differentially attractive to corn and rice strain males, and b) how the between-strain variation is related to the geographic variation in sexual communication that has been found in the past. Strain-specific lures will probably be more effective to assess the population distributions of the two strains than the commercial lures that have been used so far, e.g., [27,54-56]. If the ratios found in the glands are an indication of the ratios emitted by the females, a blend with a larger relative amount of Z11-16:Ac is likely to be more attractive to corn than to rice strain males. If $\mathrm{Z11-16:Ac}$ is less important in the attraction of rice strain males, this might explain why the addition of this compound to the two-component blend did attract more males in Mexico [27] and Costa Rica [26], but not in Florida [23] or Brazil [25]: the latter experiments may have been conducted in areas or periods when mostly rice-strain males were present.

\section{Conclusion}

The two strains of $S$. frugiperda are not only differentiated in their host use and their timing of sexual activities at night, but also in their sex pheromone composition. We found significant differences in the pheromone blend between the two strains when we considered the seven compounds that were identified from the pheromone gland of this species (see Table 1), and when we only considered the four pheromone components that have been shown to affect the attraction of $S$. frugiperda males. Even when females were injected with PBAN, which reduced the among-strain differences, the pheromone composition still significantly differed between the two strains. The pheromone composition of the hybrid females suggests a maternal inheritance of the relative amount of Z1116:Ac (m) and a genetic dominance of alleles from the corn strain in the production of 14:Ac (a), Z11-14:Ac (b), 
and Z9-12:Ac (d). The traits that differentiate the two host strains of this species (host use and differential timing of sexual activities at night) are most likely not independent of each other. For example, host plants have been found to directly or indirectly affect the sexual communication in moths [57-59]. Finding the genetic basis of both sexual (i.e. behavioral) and host plant (i.e. habitat) differentiation in this species will potentially give insight into the interaction between behavioral isolation and habitat isolation, and their role in speciation [60].

\section{Methods \\ Insects}

FAW corn and rice strains were obtained in 2006 from labreared colonies of R. Meagher at USDA-ARS in Gainesville, Fl. The corn strain was established from $>100$ larvae collected from corn plants near Homestead in MiamiDade Co., Fl, throughout October and November 2004, and was called JS3C. The rice strain colony originated from $>200$ larvae collected from pasture grasses from the Range Cattle Research and Education Centre, Ona, Hardee Co., Fl, between May 2003 and October 2003, and was named OnaR. Both colonies were reared in mass culture for 10 and 21 generations, respectively, on a pinto bean-based artificial diet at USDA Florida. In July 2006, a subset of these colonies were transferred to our laboratory. Upon receiving the colonies, 48 JS3C and 56 OnaR individuals were screened for the strain-specific COI marker. All but three JS3C individuals had the RFLP marker that is associated with the corn strain, and all OnaR individuals had the RFLP marker that is associated with the rice strain $[15,16]$. Offspring of the three ambiguous JS3C individuals were not included in subsequent rearing. Individuals used for our experiments had been reared for another 15 generations in our laboratory, in environmental chambers at $26 \pm 1{ }^{\circ} \mathrm{C}, 60 \pm 10 \% \mathrm{RH}$, and a 14:10 L:D photoperiod, in a single pair mating protocol that is specifically designed to maintain the genetic variation and to avoid selection of any females. In short, in every generation an equal number of offspring is chosen from each of 30 single pair matings. The offspring from these matings are randomly paired, again in single pairs, in the next generation to maximize effective population size and avoid possible shifts in allele frequencies. Adults used for the pheromone experiments were placed as larvae in a reversed $\mathrm{L}: \mathrm{D}$ chamber, where lights were off from $11.00-21.00 \mathrm{~h}$. Pupae were kept individually in plastic cups and checked daily for emergences. Emerged females were given a honey-water solution and left in the same rearing chamber until their glands were extracted. Pheromone glands were extracted from 2-3 day old virgin females.

\section{Gland extractions}

Two groups of glands were extracted, one to assess the between-strain variation and mode of inheritance of strain differences (Group I), and one to assess the effect of PBAN (Group II). Of group I, glands were extracted from corn and rice strain females, as well as from hybrid female offspring from crosses with corn females and rice males, referred to as $\mathrm{CxR}$, and from crosses with rice females and corn males, referred to as $\mathrm{RxC}$. To minimize the effect of one specific cross, glands were extracted from offspring of 9-10 crosses. All females were injected with Hez-PBAN (Peninsula Laboratories, San Carlos, CA) ca. 1-2 h before the scotophase. A stock solution of Hez-PBAN (200 pmol/ $\mu \mathrm{l}$ in $50 \%$ methanol and $1 \mathrm{~N} \mathrm{HCl}$ ) was diluted in saline to $3.75 \mathrm{pmol} / \mathrm{ul}$ within $1 \mathrm{hr}$ of injection. Females were injected with $2 \mu \mathrm{l}$ of this dilution, using a $10 \mu \mathrm{l}$ syringe (Hamilton, Reno, NV) with a 31 gauge needle that was inserted ventrally between the 8th and the 9th abdominal segments. All females were injected at similar times, 1-2 $\mathrm{h}$ before the scotophase, and glands were dissected 2-3 hours later. Of group II, glands were extracted either from females 4-6 h into scotophase, or from females that were injected with PBAN ca. 1-2 h before the scotophase. To assess whether possible changes in the pheromone composition was dependent on the dose of PBAN injected, rice females were injected with 2 ul of PBAN of either 0.5, 3.75 or $10 \mathrm{pmol} / \mathrm{ul}$. Because of a limited number of corn females available at the time of this experiment, corn females were injected only with 2 ul of $3.75 \mathrm{pmol} / \mathrm{ul}$ (the second dilution). Two to three hours after PBAN injection, the glands were dissected from the females.

All pheromone glands were dissected and placed in conical vials containing $50 \mu \mathrm{l}$ hexane and $40 \mathrm{ng}$ pentadecane as internal standard. After $30-40 \mathrm{~min}$, the glands were removed and the extracts were stored at $-20^{\circ} \mathrm{C}$ until analysis. The hexane extract was reduced under a gentle stream of $\mathrm{N}_{2}$ to $1-2 \mu \mathrm{l}$, taken up into $2 \mu \mathrm{l}$ octane, and placed in a $50 \mu$ l glass insert within a crimp-capped vial. Using a 7683 automatic injector, the entire volume (i.e. 3-4 $\mu \mathrm{l}$ ) of extract was injected into a splitless inlet of a HP7890 gas chromatograph (GC) coupled with a high resolution polar capillary column (DB-WAXetr [extended temperature range]; $30 \mathrm{~m} \times 0.25 \mathrm{~mm} \times 0.5 \mu \mathrm{m})$ and a flame-ionization detector (FID), programmed from $60^{\circ} \mathrm{C}$ with a 2 min hold, to $180^{\circ} \mathrm{C}$ at $30^{\circ} \mathrm{C} / \mathrm{min}$, then to $230^{\circ} \mathrm{C}$ at $5^{\circ} \mathrm{C} /$ min, during which all the pheromone components eluted. The column was then heated to $245^{\circ} \mathrm{C}$ at $20^{\circ} \mathrm{C} /$ min and held at this temperature for 15 min to clean the column before the next analysis. The FID detector was held at $250^{\circ} \mathrm{C}$.

\section{Chemical analysis}

Of all the compounds that have been previously identified from glands of $S$. frugiperda (see Table 1), we identified the following from the pheromone glands: Z9-14:Ac (M), Z11-16:Ac (m), 14:Ac (a), Z11-14:Ac (b), 12:Ac (c), Z9-12:Ac (d), and Z7-12:Ac (e). The letters behind the compound are given to facilitate the reading. All of these 
compounds were found in both strains. These compounds were first identified by injecting synthetic compounds into the GC described above to compare their retention times with the retention times of the peaks present in the gland extracts. In addition, the alcohol and some of the aldehyde analogs of these compounds (i.e., Z9-14:Ald, Z11-16:Ald, Z9-12:OH and Z7-12:Ac) were injected as well to make sure that these compounds have a different retention time in our settings and GC program. All synthetic compounds were bought from Pherobank, Wageningen. After the first identification, the chemical identities of all peaks in the gland extracts were checked by GC-MS. A subset of extracts was injected into an HP6890 GC coupled to Masspec MS002 (Micromass, Manchester, UK) with electron ionization (EI) at $70 \mathrm{eV}$, and separated using a $30 \mathrm{~m} \times 0.25 \mathrm{~mm} \times 0.25 \mu \mathrm{m}$ DB-Wax column, with the same temperature program as described above. The recorded mass spectra were compared to those of known standards injected in the same manner and using spectral database (Wiley MS library v 7). The gland extracts were additionally screened for a possible detection of E7-12:Ac. This compound was not found in the 10 extracts examined.

The amount of each pheromone compound was calculated relative to the $40 \mathrm{ng}$ internal standard. Every day, before and after each GC sequence, we injected authentic standards of the pheromone compounds mentioned above to assess column performance as well as to check the retention times of each of the components. We corrected all integration results by the differential response of the FID to the various authentic standards. Because there is high variance among female moths in total gland pheromone content, even within treatments, most researchers analyze differences between the amount of each component after converting amounts to percentages relative to the most abundant compound (i.e., the "major" component) $($ e.g. $[8,23]$. Therefore, we compared the pheromone composition between different groups of females by converting all amounts to relative percentages of the total amount of all pheromone components in the glands.

\section{Statistical analysis}

To compare the pheromone compositions between the two strains, a multivariate analysis of variance (MANOVA) was conducted, using SAS, version 9.1 (SAS Institute, 2002-2003), with all the females of a group that were a) extracted during the scotophase, or b) injected with 7.5 pmol PBAN. In addition, to assess the effect of PBAN on the pheromone composition within each strain, a separate MANOVA was conducted within each species. To determine the mode of inheritance of the difference in pheromone composition between corn and rice strain females, another MANOVA was conducted, comparing the pheromone composition between the corn strain females, the rice strain females, the CxR hybrid females, and the $\mathrm{RxC}$ hybrid females. In every test, the means of all pheromone compounds were separated using leastsquares means (LSMEANS), with a Tukey adjustment for multiple comparisons.

\section{Phenotypic correlations}

To assess possible enzymatic differences between the two strains in the biosynthetic pathway of the FAW pheromone, we analyzed phenotypic associations among pheromone components in the two strains by generating a Pearson's correlation matrix (PROC CORR in SAS). For this correlation matrix we used the percentages of each compound relative to the total amount of all the pheromone compounds that we distinguished. Separate correlation matrices were constructed for the pheromone composition found in glands of a) corn or rice females from which glands were extracted under natural conditions, i.e. in the scotophase, b) corn or rice females that were injected with 7.5 pmol PBAN, which glands were extracted at the same time as the hybrid females, and c) hybrid $\mathrm{CxR}$ or $\mathrm{RxC}$ females that were injected with 7.5 pmol PBAN as well. Since such associations can give insight into biosynthetic pathways of the pheromone components, we also constructed a (hypothetical) scheme of this pathway.

\section{Abbreviations}

The chemical compounds are abbreviated following the standard shorthand notation for pheromone molecules [61]. For example, $(Z)$-11-hexadecen-1-yl acetate is abbreviated Z11-16:Ac. The corresponding aldehyde is abbreviated Z11-16:Al. PBAN: Pheromone Biosynthesis Activating Neuropeptide.

\section{Competing interests}

The authors declare that they have no competing interests.

\section{Authors' contributions}

ATG designed the experiments. MM conducted the experiments. ATG, MM and GS analyzed the data. SL and AS carried out the chemical analyzes and identifications. ATG and DGH interpreted the data and drafted the manuscript. MM, GS, SL and AS critically revised the manuscript. All authors read and approved the final manuscript.

\section{Additional material}

\footnotetext{
Additional file 1

Pearson's correlation coefficients of pheromone compounds in glands of A) corn strain females, and B) rice strain females, extracted in scotophase. The tables show positive and negative phenotypic correlations between all pheromone compounds. The colors of the cells coincide with the colors in the proposed biosynthetic pathway of the compounds in Figure 5. Click here for file

[http://www.biomedcentral.com/content/supplementary/17429994-5-20-S1.doc]
} 


\section{Additional file 2 \\ Pearson's correlation coefficients of pheromone compounds in glands of $A$ ) PBAN-injected corn strain females, and B) PBAN-injected rice strain females. The tables show positive and negative phenotypic correlations between all pheromone compounds. The colors of the cells coincide with the colors in the proposed biosynthetic pathway of the compounds in Figure 5, while the blue cells indicate a strikingly different correlation from that found in other females. \\ Click here for file \\ [http://www.biomedcentral.com/content/supplementary/1742- 9994-5-20-S2.doc] \\ Additional file 3 \\ Pearson's correlation coefficients of the pheromone compounds in glands of A) CxR hybrid females, and B) RxC hybrid females. The tables show positive and negative phenotypic correlations between all pheromone com- pounds. The colors of the cells coincide with the colors in the proposed bio- synthetic pathway of the compounds in Figure 5, while the blue cell indicates a strikingly different correlation from that found in other females. \\ Click here for file \\ [http://www.biomedcentral.com/content/supplementary/1742- 9994-5-20-S3.doc]}

\section{Acknowledgements}

We thank Rob Meagher for providing us with corn and rice strain individuals, from which we could start our colonies. We thank Regina Seibt, Steffen Reifarth, Sabine Hänniger and Johannes Fleischmann for rearing the two strains, and Jörgen Unbehend for his help in preparing the pheromone vials. We also thank three anonymous reviewers for helpful comments on the manuscript. This research was supported by the Max-Planck-Gesellschaft.

\section{References}

I. Percy-Cunningham JE, MacDonald JA: Biology and ultrastructure of sex pheromone-producing glands. In Pheromone biochemistry Edited by: Prestwich GD, Blomquist GJ. Academic Press, Inc.: Orlando, Fl; 1987:27-69.

2. Raina AK, et al.: Structural organization of the sex pheromone gland in Helicoverpa zea in relation to pheromone production and release. Arthropod Structure \& Development 2000, 29(4):343-353.

3. Bjostad LB, Wolf WA, Roelofs WL: Pheromone biosynthesis in lepidopterans: desaturation and chain shortening. In Pheromone biochemistry Edited by: Prestwich GD, Blomquist G]. Academic Press, Inc.: Orlando, Fl; 1987:77-II 7.

4. Jurenka R: Insect pheromone biosynthesis. Chemistry of Pheromones and Other Semiochemicals I 2004:97-I3I.

5. Vetter RS, Baker TC: Behavioral-Responses of Male HeliothisVirescens in a Sustained-Flight Tunnel to Combinations of 7 Compounds Identified from Female Sex-Pheromone Glands. Journal of Chemical Ecology 1983, 9(6):747-759.

6. Cardé RT, Haynes KF: Structure of the pheromone communication channel in moths. In Advances in insect chemical ecology Edited by: Carde RT, Millar JG. Cambridge University Press: Cambridge, UK; 2004:283-332.

7. Heath RR, et al.: Periodicity of female sex pheromone titer and release in Heliothis subflexa and $H$. virescens (Lepidoptera, Noctuidae). Annals of the Entomological Society of America 199I, 84(2): 182-189.

8. El-Sayed AM: The Pherobase: Database of insect pheromones and semiochemicals. 2008.

9. Butlin R, Trickett AJ: Can population genetic simulations help to interpret pheromone evolution? In Insect pheromone research: New directions Edited by: Cardé RT, Minks AK. Chapman and Hall: New York; 1997:548-562.
10. Lofstedt C: Moth pheromone genetics and evolution. Philosophical Transactions of the Royal Society of London Series B-Biological Sciences 1993, 340( I 292): 167-I77.

II. Coyne JA, Orr HA: Speciation. Sunderland, MA: Sinauer Associates, Inc; 2004:545.

12. Pashley DP: Host-associated genetic differentiation in Fall armyworm (Lepidoptera, Noctuidae) - a sibling species complex. Annals of the Entomological Society of America 1986, 79(6):898-904.

13. Pashley DP, Johnson SJ, Sparks AN: Genetic population structure of migratory moths - the Fall armyworm (Lepidoptera, Noctuidae). Annals of the Entomological Society of America 1985, 78(6):756-762.

14. Lu YJ, Adang MJ: Distinguishing fall armyworm (Lepidoptera: Noctuidae) strains using a diagnostic mitochondrial DNA marker. Florida Entomologist 1996, 79(1):48-55.

15. Levy HC, Garcia-Maruniak A, Maruniak JE: Strain identification of Spodoptera frugiperda (Lepidoptera : Noctuidae) insects and cell line: PCR-RFLP of cytochrome oxidase $\mathbf{C}$ subunit I gene. Florida Entomologist 2002, 85(I): 186-190.

16. Nagoshi RN, et al.: New restriction fragment length polymorphisms in the cytochrome oxidase I gene facilitate host strain identification of fall armyworm (Lepidoptera : Noctuidae) populations in the southeastern United States. Journal of Economic Entomology 2006, 99(3):67I-677.

17. Pashley DP, Ke LD: Sequence evolution in mitochondrial ribosomal and ND-I genes in Lepidoptera - Implications for phylogenetic analysis. Molecular Biology and Evolution 1992, 9(6): $106 \mid-1075$.

18. Prowell DP, McMichael M, Silvain JF: Multilocus genetic analysis of host use, introgression, and speciation in host strains of fall armyworm (Lepidoptera : Noctuidae). Annals of the Entomological Society of America 2004, 97(5): 1034- 1044.

19. McMichael M, Prowell DP: Differences in amplified fragmentlength polymorphisms in fall armyworm (Lepidoptera : Noctuidae) host strains. Annals of the Entomological Society of America 1999, 92(2): |75-|8|.

20. Nagoshi RN, Meagher RL: FR tandem-repeat sequence in fall army-worm (Lepidoptera : Noctuidae) host strains. Annals of the Entomological Society of America 2003, 96(3):329-335.

21. Pashley DP, Hammond AM, Hardy TN: Reproductive isolating mechanisms in Fall armyworm host strains (Lepidoptera, Noctuidae). Annals of the Entomological Society of America 1992, 85(4):400-405.

22. Schöfl G, Heckel DG, Groot AT: Time-shifted reproductivebehaviours among fall armyworm (Spodoptera frugiperda) host races:Evidence for varying modes of inheritance. 2008 in press.

23. Tumlinson JH, et al:: Sex pheromone of Fall armyworm, Spodoptera frugiperda Smith, J.E. - Identification of components critical to attraction in the field. Journal of Chemical Ecology 1986, 12(9): 1909-1926.

24. Descoins C, et al.: Monitoring of crop pests by sexual trapping of males in the French West-Indies and Guyana. Agriculture Ecosystems \& Environment 1988, 21(I-2):53-65.

25. Batista-Pereira LG, et al.: Isolation, identification, synthesis, and field evaluation of the sex pheromone of the Brazilian population of Spodoptera frugiperda. Journal of Chemical Ecology 2006, 32(5): 1085-1099.

26. Andrade R, Rodriguez C, Oehlschlager AC: Optimization of a pheromone lure for Spodoptera frugiperda (Smith) in Central America. Journal of the Brazilian Chemical Society 2000, I I (6):609-613.

27. Malo EA, et al.: Evaluation of commercial pheromone lures and traps for monitoring male fall armyworm (Lepidoptera : Noctuidae) in the coastal region of Chiapas, Mexico. Florida Entomologist 200I, 84(4):659-664.

28. Mitchell ER, Tumlinson JH, McNeil JN: Field evaluation of commercial pheromone formulations and traps using a more effective sex pheromone blend for the Fall armyworm (Lepidoptera, Noctuidae). Journal of Economic Entomology 1985, 78(6): 1364-1369.

29. Fleischer SJ, et al.: Spodoptera frugiperda pheromone lures to avoid nontarget captures of Leucania phragmatidicola. J Econ Entomol 2005, 98(I):66-7I. 
30. El-Sayed AM, et al.: Geographic variation in pheromone chemistry, antennal electrophysiology, and pheromone-mediated trap catches of North American populations of the obliquebanded leafroller. Environmental Entomology 2003, 32:470-476.

31. Gemeno C, Lutfallah AF, Haynes KF: Pheromone blend variation and cross-attraction among populations of the black cutworm moth (Lepidoptera: Noctuidae). Annals of the Entomological Society of America 2000, 93: $1322-1328$.

32. Tóth M, et al.: Attraction of male Turnip moths Agrotis segetum (Lepidoptera: Noctuidae) to sex pheromone components and their mixtures at II sites in Europe, Asia, and Africa. Journal of Chemical Ecology 1992, 18:1337-1347.

33. Busato GR, et al:: Analysis of the molecular structure and diversity of Spodoptera frugiperda (JE smith) (Lepidoptera : Noctuidae) populations associated to the corn and rice crops in Rio Grande do Sul State, Brazil. Neotropical Entomology 2004, 33(6):709-7। 6.

34. Tamaki Y: Sex pheromones. In Comprehensive insect physiology, biochemistry and pharmacology Edited by: Kerkut GA, Gilbert LI. Pergamon: New York; 1985: I45-191.

35. Raina AK, et al.: Identification of a neuropeptide hormone that regulates sex pheromone production in female moths. Science 1989, 244(4906):796-798.

36. Rafaeli A, et al:: Spatial and temporal distribution of Pheromone Biosynthesis Activating Neuropeptide in Helicoverpa (Heliothis) armigera using Ria and in-vitro bioassay. Arch Insect Biochem Physiol I99|, 18(2): II 19-129.

37. Raina AK, Davis JC, Stadelbacher EA: Sex pheromone production and calling in Helicoverpa zea (Lepidoptera, Noctuidae) effect of temperature and light. Environmental Entomology I991, 20(5): $|45|-\mid 456$.

38. Rafaeli A, Soroker V: Influence of diel rhythm and brain hormone on pheromone production in 2 lepidopteran species. Journal of Chemical Ecology 1989, I 5(2):447-455.

39. Raina AK, et al.: Characteristics of a neurohormone that controls sex pheromone production in Heliothis zea. Journal of Insect Physiology 1987, 33( I I):809-8I4.

40. Raina AK, Kempe TG, Jaffe $\mathrm{H}$ : Pheromone Biosynthesis Activating Neuropeptide - Regulation of pheromone production in moths. Acs Symposium Series 1991, 453:100-109.

41. Rafaeli A: Neuroendocrine control of pheromone biosynthesis in moths. International Review of Cytology - a Survey of Cell Biology 2002, 213:49-91.

42. Groot AT, et al.: Effect of PBAN on pheromone production by mated Heliothis virescens and Heliothis subflexa females. I Chem Ecol 2005, 3 I (I): I 4-28.

43. Rafaeli A: Mechanisms involved in the control of pheromone production in female moths: recent developments. Entomologia Experimentalis Et Applicata 2005, I I 5(1):7-15.

44. Rafaeli A, Jurenka R: PBAN regulation of pheromone biosynthesis in female moths. In Pheromone biochemistry and molecular biology Edited by: Blomquist G], Vogt R. Elsevier Academic Press: London, UK; 2003:107-I36.

45. Tillman JA, et al.: Insect pheromones - an overview of biosynthesis and endocrine regulation. Insect Biochemistry and Molecular Biology 1999, 29(6):48|-5|4.

46. Tsfadia $O$, et al.: Pheromone biosynthetic pathways: PBANregulated rate-limiting steps and differential expression of desaturase genes in moth species. Insect Biochem Mol Biol 2008, 38(5):552-567.

47. Fabrias G, Marco MP, Camps F: Effect of the Pheromone Biosynthesis Activating Neuropeptide on sex pheromone biosynthesis in Spodoptera littoralis isolated glands. Archives of Insect Biochemistry and Physiology 1994, 27(2):77-87.

48. Martinez T, Fabrias G, Camps F: Sex pheromone biosynthetic pathway in Spodoptera littoralis and its activation by a neurohormone. Journal of Biological Chemistry 1990, 265(3): | 38|- 387.

49. Jurenka R: Biochemistry of female moth sex pheromones. In Insect pheromone biochemistry and molecular biology Edited by: Blomquist G], Vogt R. Elsevier Academic Press: London, UK; 2003:53-80.

50. Rosenfield $\mathrm{CL}$, et al.: Structural and functional conservation and divergence among acyl-CoA desaturases of two noctuid species, the corn earworm, Helicoverpa zea, and the cabbage looper, Trichoplusia ni. Insect Biochem Mol Biol 200I, 3I(10):949-964
51. Groot AT, et al.: QTL analysis of sex pheromoneblend differences between two closely related moths: insights intodivergence in biosynthetic pathways. 2008 in press.

52. Knipple DC, et al:: Evolution of the integral membrane desaturase gene family in moths and flies. Genetics 2002, 162(4): 1737-1752.

53. Rodriguez $S$, et al.: Expression and evolution of Delta(9) and Delta(II) desaturase genes in the moth Spodoptera littoralis. Insect Biochemistry and Molecular Biology 2004, 34( ( 2): I | I 5- I 328

54. Mitchell ER, et al.: Seasonal periodicity of Fall armyworm, (Lepidoptera, Noctuidae) in the Caribbean basin and northward to Canada. Journal of Entomological Science I991, 26(I):39-50.

55. Nagoshi RN, Meagher RL: Behavior and distribution of the two fall armyworm host strains in Florida. Florida Entomologist 2004, 87(4):440-449.

56. Meagher RL: Trapping fall armyworm (Lepidoptera : Noctuidae) adults in traps baited with pheromone and a synthetic floral volatile compound. Florida Entomologist 200I, 84(2):288-292.

57. McNeil JN, Delisle J: Are host plants important in pheromonemediated mating systems of Lepidoptera? Experientia 1989, 45(3):236-240.

58. Landolt PJ, Phillips TW: Host plant influences on sex pheromone behavior of phytophagous insects. Annual Review of Entomology 1997, 42:37I-39I.

59. Reddy GVP, Guerrero A: Interactions of insect pheromones and plant semiochemicals. Trends in Plant Science 2004, 9(5):253-261.

60. Smadja C, Butlin RK: On the scent of speciation: the chemosensory system and its role in premating isolation. Heredity 2008. 10.1 038/hdy.2008.55

61. Roelofs WL, Hill A, Carde RT: Sex pheromone components of the redbanded leafroller, Argyroaenia velutinana. J Biol Chem 1975, I( I 0):83-89.
Publish with Biomed Central and every scientist can read your work free of charge

"BioMed Central will be the most significant development for disseminating the results of biomedical research in our lifetime. "

Sir Paul Nurse, Cancer Research UK

Your research papers will be:

- available free of charge to the entire biomedical community

- peer reviewed and published immediately upon acceptance

- cited in PubMed and archived on PubMed Central

- yours - you keep the copyright
BioMedcentral 